\title{
Breast Epithelial-myoepithelial Carcinoma With HER2 Gene Amplification: a Rare Case and Review of Literature
}

\section{Min $\mathrm{Hu}$}

Hubei Cancer Institute: Hubei Cancer Hospital

Junqiu Yue

Hubei Cancer Institute: Hubei Cancer Hospital

\section{Fang Guo}

Hubei Cancer Institute: Hubei Cancer Hospital

Su Jin

Hubei Cancer Institute: Hubei Cancer Hospital

Mingwei Wang

Hubei Cancer Institute: Hubei Cancer Hospital

Na Fang ( $053651127 @ q q . c o m$ )

Hubei Cancer Institute: Hubei Cancer Hospital

\section{Case Report}

Keywords: adenomyoepithelioma, epithelial-myoepithelial carcinoma, HER2, fluorescence in situ hybridization

Posted Date: June 29th, 2021

DOI: https://doi.org/10.21203/rs.3.rs-659171/v1

License: (c) (1) This work is licensed under a Creative Commons Attribution 4.0 International License. Read Full License 


\section{Abstract}

Background: Epithelial-myoepithelial carcinoma is a very rare carcinoma that both luminal and myoepithelial components are malignant. What's more, HER2 gene amplification has not been detected in the literature up to now. Herein, we report a rare case of breast epithelial-myoepithelial carcinoma with HER2 gene amplification.

Case presentation: An 80-year-old woman presented with a mass in the upper outside region of her left breast. The core needle biopsy of the left breast mass revealed invasive breast carcinoma, and then modified radical mastectomy of left breast was performed. Macroscopically, the tumor was measured $6 \mathrm{~cm}$ in diameter, and it was a solid and lobulated mass with areas of cystic and hemorrhagic lesions. Microscopically, the histological findings of tumor were consisted of 3 components. One component showed biphasic proliferation of both eosinophilic luminal epithelial and pale abluminal myoepithelial cells, arranging predominantly in dilated tubular architecture, which accounted for most of the lesion. Papillary architecture and eosinophilic secretions could be seen in some dilated ducts. The second component was proliferation of myoepithelial cells with pale cytoplasm around the glandular epithelium forming casing-like structures. The third component was few solid-appearing areas which was displayed a predominance of monophasic proliferation of myoepithelial cells. Both cell types exhibited enlarged and markedly atypical nuclei, with obvious nucleoli and prominent mitoses (up to 10-15/10 high power fields). Foci of infiltration were identified at the periphery of the lobules and the surrounding adipose tissue. Areas of central necrosis and stromal hyalinization could be seen. A diagnosis of EMC was made by morphology and immunohistochemistry. It's worth noting that HER2 gene amplification has not been detected in malignant adenomyoepithelioma in the literature up to now; While in our present report, HER2 was $2+$ membranous immunoreactivity, and then HER2 gene amplification was detected by fluorescence in situ hybridization.

Conclusion: Accurate diagnosis of malignant adenomyoepithelioma is challenging but of great importance, and the management of malignant adenomyoepithelioma would be made according to histologic features.

\section{Introduction}

Adenomyoepithelioma (AME) is a rare tumor characterized by biphasic neoplastic proliferation of the luminal glandular epithelial and abluminal myoepithelial cells of the breast [1]. One or both of the components of the tumor may exhibit malignant transformation, which is called malignant adenomyoepithelioma (AME-M). According to the 5th World Health Organization (WHO) classification of tumors of the breast [2], AME-M is AME with carcinoma, in which the malignancy may arise from either luminal epithelial or myoepithelia components, or from both cell types. When both epithelial and myoepithelial compartments are malignant, the term "epithelial-myoepithelial carcinoma" (EMC) is used. Accurate diagnose of EMC remains challenging because of the rarity of the disease. What's more, human 
epidermal growth factor receptor 2 (HER2) gene amplification has not been detected in the literature up to now [3]. Herein, we report a rare case of breast EMC with HER2 gene amplification.

\section{Case Presentation}

An 80-year-old female presented with a mass in the upper outside region of her left breast, which had been observed at local clinic for more than one year. The size of the mass was about $5 \mathrm{~cm} \times 3 \mathrm{~cm}$, and it was palpable hard, smooth and painless. Mammography showed lump-like mass shadow on the outside of the left breast with unclear boundary and uneven density, corresponding to BI-RADS (Breast Imaging Reporting and Data System) category IVc (Fig. 1A). The core needle biopsy of the left breast mass revealed invasive carcinoma, and left mastectomy with axillary lymph node dissection was performed.

Macroscopically, the tumor was measured $6 \mathrm{~cm}$ in diameter and it was a solid lobulated mass with areas of cystic and hemorrhagic lesions (Fig. 1B). Microscopically, the histological findings of tumor were consisted of 3 components. One component showed biphasic proliferation of both eosinophilic luminal epithelial and pale abluminal myoepithelial cells, arranging predominantly in dilated tubular architecture, which accounted for most of the lesion. Papillary architecture and eosinophilic secretions could be seen in some of the dilated ducts (Fig. 1C). The second component was proliferation of myoepithelial cells with pale cytoplasm around the glandular epithelium forming casing-like structures (Fig. 1D). The third component was few solid-appearing areas displayed a predominance of monophasic proliferation of myoepithelial cells (Fig. 1E). The nuclei of both cells types were large and markedly atypical with thickened nuclear membrane, hyperchromatic nuclei, obvious nucleoli, and prominent mitoses (up to 10$15 / 10$ high power fields). Foci of infiltration were identified at the periphery of the lobules and the surrounding adipose tissue. Areas of central necrosis and stromal hyalinization could be seen.

By immunohistochemistry $(\mathrm{IHC})$, in areas with a biphasic proliferation of epithelial and myoepithelial cells forming dilated tubular architecture (Fig. 2A), both biomarkers for epithelial cells (Fig. 2B) and myoepithelial cells were positive (Fig. 2C). So was in casing-like structures (Fig. 2D-F). In solid-appearing areas (Fig. 2G), biomarkers for epithelial cells were weak positive (Fig. 2H), while biomarkers for myoepithelial cells were strong positive (Fig. 2I). Ki-67 labeling index (Li) was 30\%. Estrogen receptor (ER) and progesterone receptor (PR) were nagative; HER2 in epithelial cells was $2+$ membranous immunoreactivity (Fig. 3A). Then HER2 gene amplification was detected by fluorescence in situ hybridization (FISH) (Fig. 3B). A diagnosis of epithelial-myoepithelial carcinoma was made. Neither sentinel nor axillary lymph nodes showed metastases. The patient did not receive anti-HER2 targeted therapy or any adjuvant systemic therapy for personal reasons. She has no complications or recurrences at present.

\section{Discussion}

AME is a biphasic neoplasm (usually benign) characterized by small epithelium-lined spaces with inner luminal ductal cells and a proliferation of variably enlarged and cleared noticeable abluminal 
myoepithelial cells. Rarely, one or both components of AME can undergo a malignant transformation called AME-M. However, the criteria for malignant transformation of AME have not been well established. Malignant features in AME-M reported by literatures including cytological atypia, infiltrative growth, necrosis and increased mitotic figures ( $>3 / 10 \mathrm{HPF}$ ) [4]. The malignant transformation can present a wide spectrum of morphological features, such as features of invasive breast carcinoma of no special type, lobular carcinoma, metaplastic carcinoma, and myoepithelial cell carcinoma [5]. Nevertheless, the histological morphology of biphasic proliferation and the malignant biological behavior are the key points for the differential diagnosis.

In the 5th WHO classification of tumors of the breast, AME-M is divided into AME with carcinoma and EMC [2]. EMC is a very rare carcinoma that both luminal and myoepithelial components are malignant. EMCs are multilobulated tumors with pushing or infiltrative margins, displaying biphasic carcinomas composed of a mixture of malignant epithelial and myoepithelial cells. Immunohistochemical characteristic is biphasic, with luminal epithelial cells expressing CK7 and EMA; and abluminal myoepithelial cells expressing markers like SMA, SMMHC, CD10, p63, Calponin and CK5/6 [6].

In this report, we described a case of EMC of the breast, which mainly consisted of three typical structural features. One component was biphasic proliferation of both luminal epithelial and abluminal myoepithelial cells, arranging predominantly in dilated tubular architecture; Papillary architecture and eosinophilic secretions could be commonly seen in some dilated ducts. The second component was predominant proliferation of myoepithelial cells surrounding or compressing the glandular structures lined by epithelial cells. Both cell types exhibited markedly cellular atypia, supporting a malignant transformation. These biphasic proliferation of both luminal epithelial and abluminal myoepithelial cells which were positive for both biomarkers of epithelial cells and myoepithelial cells, could be differentiated from invasive ductal carcinoma. The third component was few solid-appearing areas displayed a predominance of monophasic proliferation of myoepithelial cells, and biomarkers for myoepithelial cells were positive. This should be differentiated from myoepithelial carcinoma and metaplastic carcinoma. Widely sampled for background or areas of AME is a good method.

Genetic analyses suggested that AME-M might arise from ER-negative AME, which demonstrating no HER2 gene amplification [3]. However, AME-M especially EMC might arise irrespective of the presence of AME. The status of HER2 gene in AME-M was negative in almost all of the previous reports, except one case by Sugano et al. [7]. They reported two cases of AME-M that both cases were ER negative, while HER2 oncoprotein was $2+$ in one of the cases. The same was found in our present case that HER2 protein was $2+$ by IHC; Then we performed HER2 FISH and demonstrated HER2 gene amplification. However, the patient did not receive anti-HER2 targeted therapy for personal reasons.

For patients with AME-M, haematogenous spread is more frequently than lymphatic spread, and the need for axillary dissection is unknown [8]. What's more, the need for adjuvant therapy including chemotherapy and/or radiotherapy also remains unclear [9]. In our present case, the patient underwent both sentinel and axillary lymph nodes dissection, and did not observe any lymph node involvement. She did not receive 
any adjuvant systemic therapy as well. At present, she has no complications or recurrences three months after surgery. We will continue to follow up.

\section{Conclusion}

AME-M is a very rare breast tumor which has various morphological features. Due to the rarity of AME-M, there are no standard treatment guidelines currently. Thus, an accurate diagnosis of AME-M is challenging but of great importance, and the management of AME-M would be made according to histologic features. Furthermore, molecular studies and larger case series are needed to clarify HER2 amplification or not in AME-M.

\section{Abbreviations}

AME: Adenomyoepithelioma; AME-M: Malignant adenomyoepithelioma; EMC: epithelial-myoepithelial carcinoma; HER2: Human epidermal growth factor receptor 2; BI-RADS: Breast Imaging Reporting and Data System; IHC: Immunohistochemistry; ER: Estrogen receptor; PR: Progesterone receptor; FISH:

Fluorescence in situ hybridization.

\section{Declarations}

\section{Acknowledgements}

Not applicable.

\section{Authors' contributions}

The manuscript was drafted by Min Hu and Na Fang; Na Fang supervised the preparation of this case report; Su Jin and Mingwei Wang performed the research; Junqiu Yue and Fang Guo assessed the results. All authors have read and approved the final manuscript.

\section{Funding}

No funding.

\section{Availability of data and materials}

The dataset supporting the conclusions of this article is included within the article.

\section{Ethics approval and consent to participate}

All ethical approval and consent procedures were approved by the Medical Ethical Committee of Hubei Cancer Hospital, Tongji Medical College of Huazhong University of Science and Technology. According to the institution guidelines, a single case report with de-identified patient specific information, the patient's singed privacy consent is not necessary. 


\section{Consent for publication}

Not applicable.

\section{Competing interests}

The authors declare that there are no potential conflicts of interest with respect to authorship, and/or publication of this article.

\section{References}

1. Hamperl $\mathrm{H}$. The myothelia (myoepithelial cells). Normal state, regressive changes, hyperplasia, tumors[ J]. Curr Top Pathol.1970,53:161 -220.

2. WHO Classification of Tumors Editorial Board, ed. WHO classification of tumors, 5th edition- Breast tumors. Lyon: International Agency for Research on Cancer 2019.

3. Felipe C Geyer, Anqi Li, Anastasios D Papanastasiou, et al. Recurrent hotspot mutations in HRAS Q61 and PI3K-AKT pathway genes as drivers of breast adenomyoepitheliomas. Nat Commun. 2018; 9(1): 1816.

4. Hayes MM. Adenomyoepithelioma of the breast: a review stressing its propensity for malignant transformation. J Clin Pathol 2011;64:477-84.

5. Moritz AW, Wiedenhoefer JF, Profit AP, et al. Breast adenomyoepithelioma and adenomyoepithelioma with carcinoma (malignant adenomyoepithelioma) with associated breast malignancies: a case series emphasizing histologic, radiologic, and clinical correlation. Breast 2016;29:132-9.

6. Aanchal Kakkar, Kirti Jangra, Navin Kumar, et al. Epithelial-myoepithelial carcinoma of the breast: A rare type of malignant adenomyoepithelioma. Breast J. 2019; 25(6): 1273-1275.

7. Sugano I, Nagao T, Tajima Y, et al. Malignant adenomyoepithelioma of the breast: a non-tubular and matrix-producing variant. Pathol Int 2001; 51: 193-9.

8. Naomi Wiens, Daniel I Hoffman, Cassie Ye Huang, et al. Clinical characteristics and outcomes of benign, atypical, and malignant breast adenomyoepithelioma: a single institution's experience. Am J Surg. 2020; 219(4): 651-654.

9. Mi Jin Kim, Cheol Seung Kim, Myoug Jin Ju, et al. Malignant adenomyoepithelioma of the breast: A rare case report. Int J Surg Case Rep. 2019; 59: 111-114.

\section{Figures}



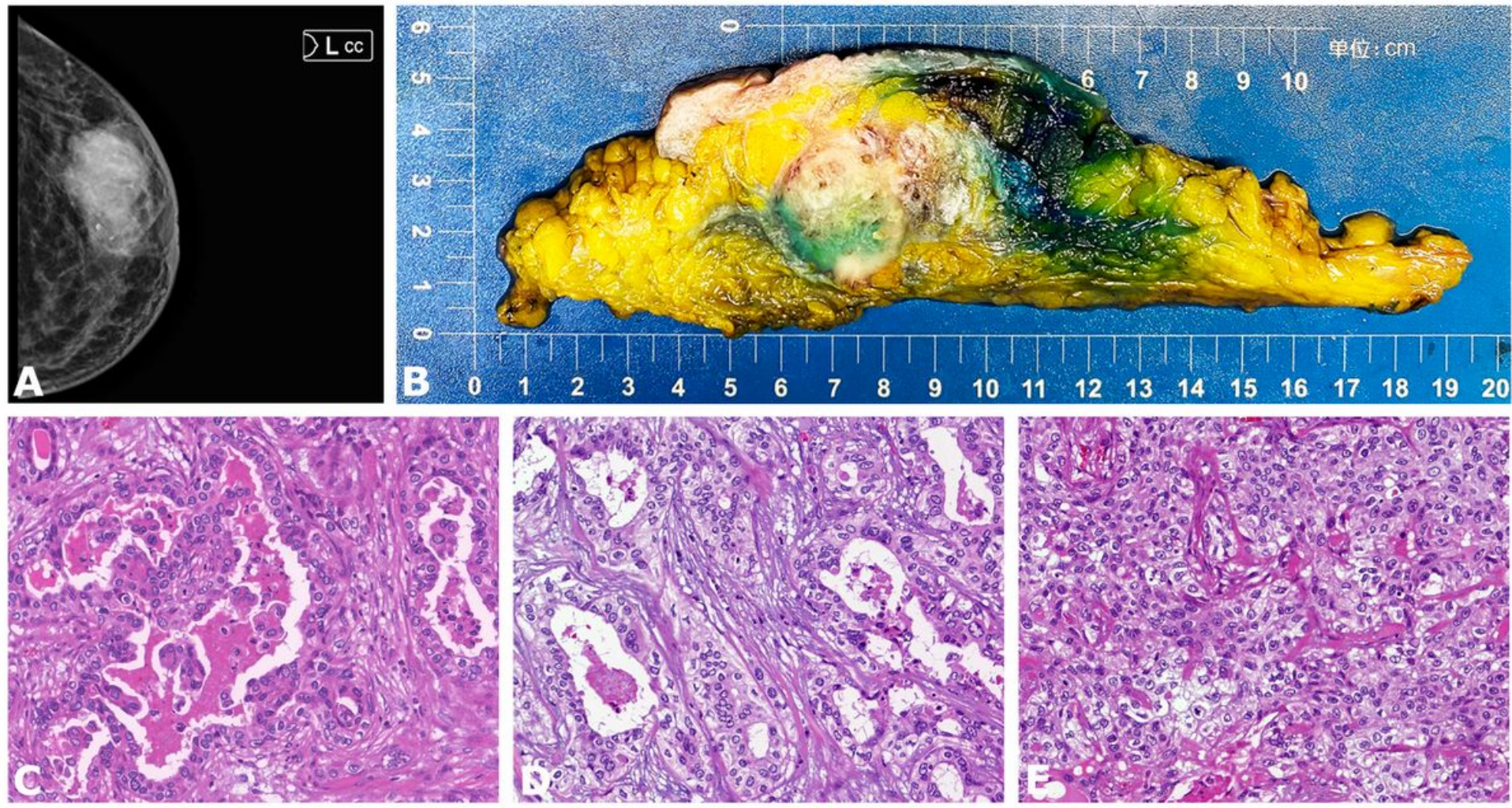

\section{Figure 1}

Mammography showing lump-like mass shadow on the outside of the left breast with unclear boundary and uneven density (A); A gross view of the tumor showed a solid lobulated mass with areas of cystic and hemorrhagic lesions (B); The area showed biphasic appearance with tubules lined by luminal and abluminal cells, papillary architecture could be seen $(C, \times 200)$; The area showed proliferation of myoepithelial cells around the glandular epithelium forming casing-like structures $(D, \times 200)$; The solidappearing areas displayed a predominance of myoepithelial cells $(E, \times 200)$. 
HE

CK7
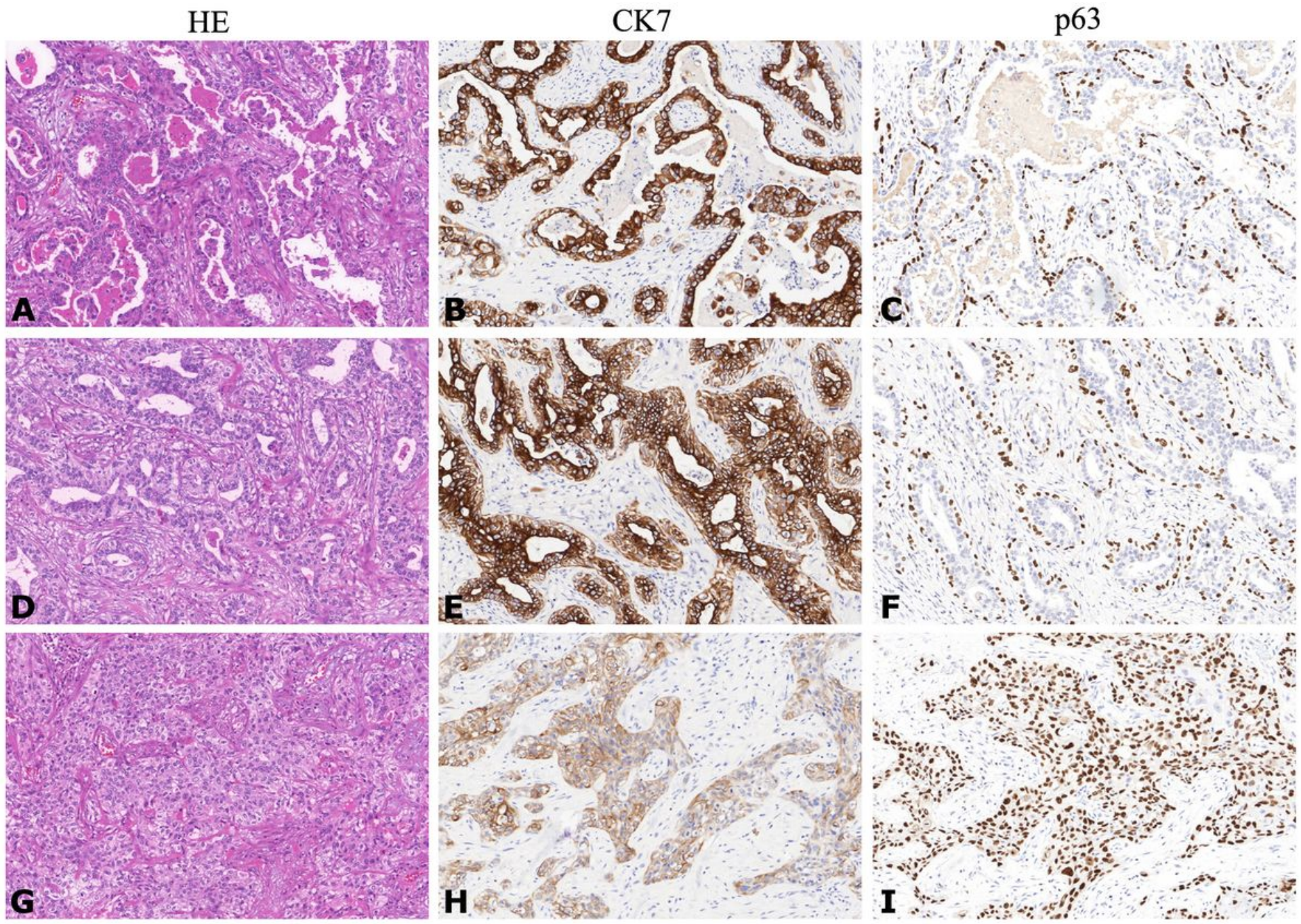

Figure 2

Areas with a biphasic proliferation of epithelial and myoepithelial cells forming dilated tubular architecture $(A, \times 100)$, both $C K 7(B, \times 100)$ and p63 staining were positive $(C, \times 100)$. Both CK7 $(E, \times 100)$ and p63 staining were positive $(F, \times 100)$ in casing-like areas $(D, \times 100)$. In solid-appearing areas $(G, \times 100)$, CK7 were weak positive $(H, \times 100)$, while biomarkers for myoepithelial cells were strong positive $(I, \times 100)$. 


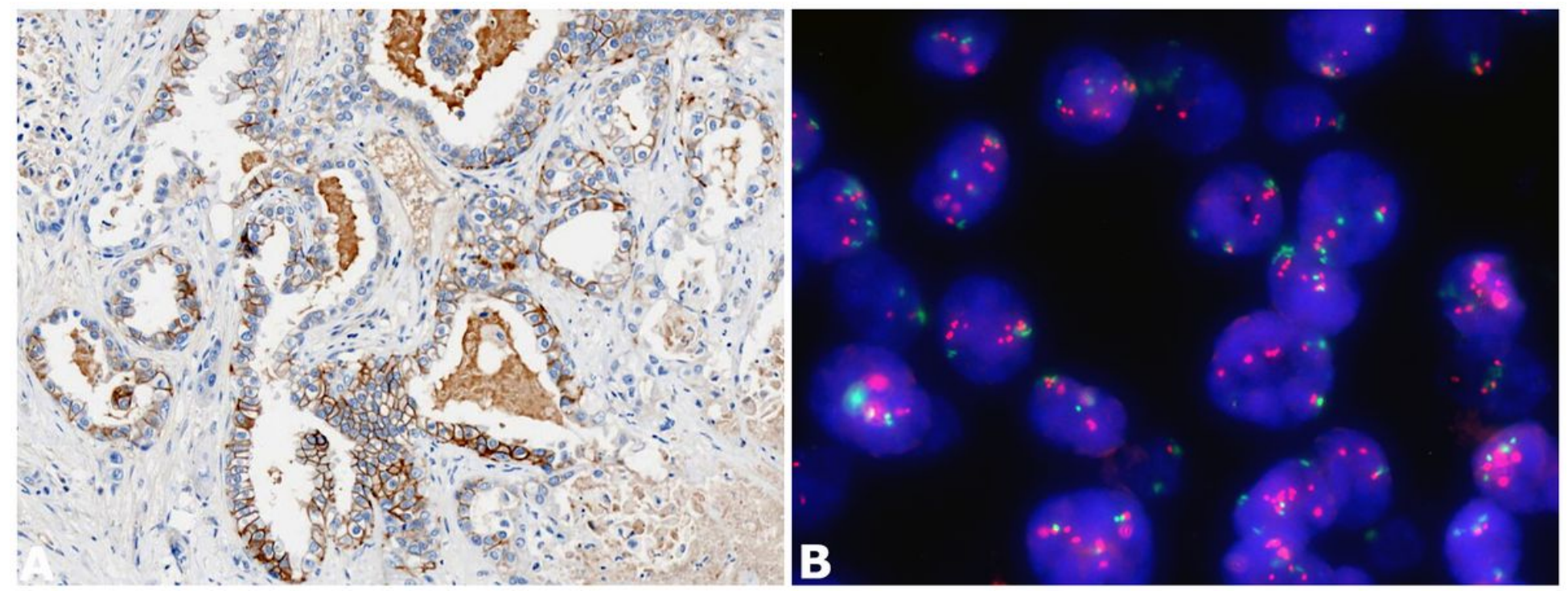

Figure 3

HER2 immunoreactivity was moderate positive (2+) in the tubular epithelial cells $(A, \times 100)$; HER2 gene amplification showed by FISH (B).

\section{Supplementary Files}

This is a list of supplementary files associated with this preprint. Click to download.

- CAREchecklist.jpg 